\title{
Collisionless shocks and particle acceleration: Lessons from studies of heliospheric shocks
}

\author{
Toshio Terasawa ${ }^{1}$ \\ ${ }^{1}$ Institute for Cosmic Ray Research, University of Tokyo, \\ 5-1-5 Kashiwa-no-ha, Kashiwa, Chiba 277-8582, Japan \\ email: terasawa@icrr.u-tokyo.ac.jp
}

\begin{abstract}
Acceleration processes at astrophysical collisionless shocks are reviewed with a special emphasis on the importance of in situ observations of heliospheric shocks. Topics to be included are nonlinear reaction of shock acceleration process, effect of neutral particles, and electron acceleration.
\end{abstract}

Keywords. heliosphere, diffusive shock acceleration, cosmic rays, nonlinearity, supernova remnants, whistler waves

\section{Introduction}

Astrophysical collisionless shock waves are thought to be efficient accelerators for nonthermal particles. In 1977-1978 Axford et al. (1977), Bell (1978), Blandford \& Ostriker (1978), and Krymsky (1977) published the idea of 'diffusive shock acceleration' (DSA) process, where nonthermal particles, cosmic rays particles for their discussions, are accelerated around shock fronts through the scattering processes repeating to occur both in the shock upstream and downstream regions. While shocks within the heliosphere largely differ from astrophysical shocks in their speeds or spatial scales, there are certain commonalities among them in basic physical mechanisms, so that our understanding of heliospheric shocks based on in situ observations could lead to comprehensive understanding of physical processes of distant astrophysical shocks.

In the heliosphere shocks are formed ahead of coronal mass ejections (CME), ahead of planetary/cometary magnetospheres/ionospheres, around corotating interaction regions (CIR), and ahead of the heliopause (see, e.g., Schwartz, 2006; Terasawa, 2011). While the usual coverage of acceleration parameters obtainable from these heliospheric observations is rather limited, we could have some exceptional values at least a few times per solar cycle. For example, while velocities of heliospheric shocks are usually of the order of several hundred $\mathrm{km} / \mathrm{s}$ or less, the historically fastest record of interplanetary shock velocities reached $\sim 4000 \mathrm{~km} / \mathrm{s}$ (Figure 1a) which is almost comparable to the shock velocities found around young supernova remnants.

Hillas (1984) argued that the maximum energy $E_{\max }$ of particles of charge $Z e$ accelerated in the region with a characteristic velocity $v$, a magnetic field $B$, and a spatial size $L$, is regulated by a simple relation,

$$
E_{\max }=Z e v B L
$$

and applied (1.1) to search possible astrophysical sites for the acceleration of ultra high energy cosmic rays $\left(E_{\max }>\sim 10^{19} \mathrm{eV}\right)$. In Fig. 1 b and Table 1 , we compare the results of (1.1) with the observed maximum energies in several heliospheric acceleration phenomena, for which we include not only due to shock acceleration but also second-order stochastic acceleration as well as reconnection-related acceleration. As seen in Fig. 1b, 
(a)

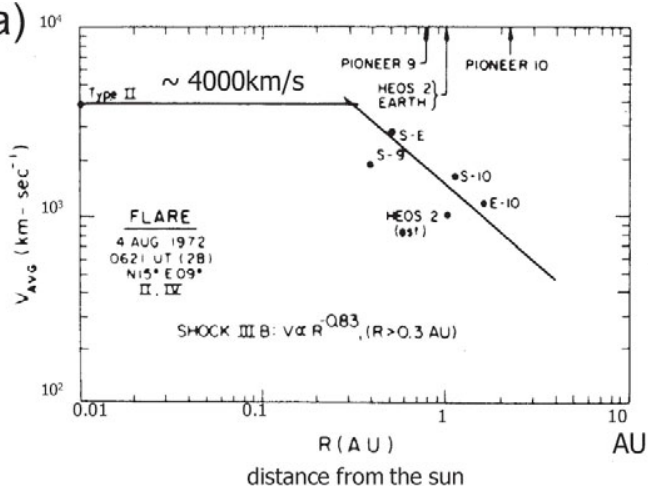

(b)

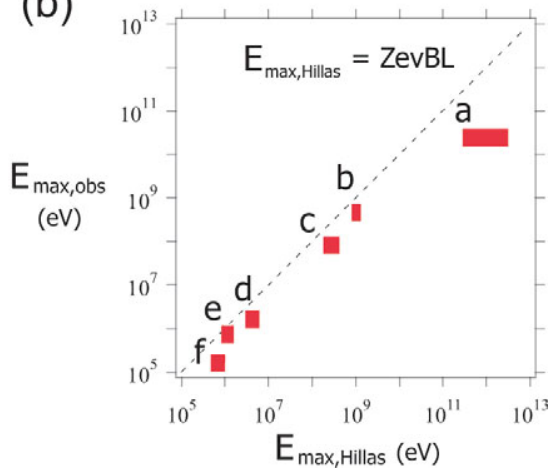

Figure 1. (a) left: The historically fastest interplanetary shock observed on 4 August 1972 (from Smart \& Shea (1985)). The horizontal and vertical axes show the distance from the sun and the estimated shock velocity, respectively. (b) right: Heliospheric Hillas relation. The horizontal and vertical axes show $E_{\mathrm{max}}$ expected from the Hillas relation (1.1) and $E_{\mathrm{max}}$ from observations, respectively. For the items, a-f, see corresponding lines in Table 1.

Table 1. Heliospheric Hillas relation vs. Observations (Terasawa, 2001))

\begin{tabular}{|c|c|c|c|c|c|c|}
\hline & sites & $B_{1} \equiv B / 1 \mathrm{G}$ & $\begin{array}{c}L_{10} \\
\equiv L / 10^{10} \mathrm{~cm}\end{array}$ & $v / c$ & $\begin{array}{c}E_{\mathrm{max}} \text { by Hillas } \\
\text { relation }(1.1)\end{array}$ & observed $E_{\mathrm{max}}$ \\
\hline $\mathrm{a}$ & $\begin{array}{c}\text { solar flares } \\
\left(\mathrm{CME}^{1} \text { shocks }\right)\end{array}$ & $10^{2}$ & 1 & 0.001-0.01 & $300 \mathrm{GeV}-3 \mathrm{TeV}$ & $\mathrm{p}<\sim 30 \mathrm{GeV}$ \\
\hline $\mathrm{b}$ & $\begin{array}{c}\text { Van Allen Belt }{ }^{2} \\
\text { earth } \\
\text { Jupiter }\end{array}$ & $\begin{array}{c}0.1 \\
1\end{array}$ & $\begin{array}{l}0.1\left(1.4 \mathrm{R}_{\mathrm{E}}\right) \\
1\left(1.4 \mathrm{R}_{\mathrm{J}}\right)\end{array}$ & $\begin{array}{l}0.03 \\
0.03\end{array}$ & $\begin{array}{c}1 \mathrm{GeV} \\
100 \mathrm{GeV}\end{array}$ & $\begin{array}{c}\mathrm{p} \sim 0.6 \mathrm{GeV} \\
?\end{array}$ \\
\hline $\mathrm{c}$ & $\begin{array}{l}\text { heliospheric shocks } \\
<\sim 1 \mathrm{AU}: B \propto L^{-2} \\
>\sim 1 \mathrm{AU}: B \propto L^{-1}\end{array}$ & $\begin{array}{l}6 \times 10^{-5} \\
\text { at } 1 \mathrm{AU}\end{array}$ & $10^{3}(1 \mathrm{AU})$ & 0.001-0.002 & $0.2-0.4 \mathrm{GeV}$ & $\begin{aligned} \mathrm{ESP}^{3} & <\sim 0.1 \mathrm{GeV} \\
\mathrm{ACR}^{4} & <\sim 0.1 \mathrm{GeV}\end{aligned}$ \\
\hline $\mathrm{d}$ & $\begin{array}{c}\text { earth's bow shock } \\
+ \\
\mathrm{IMF}^{5} \mathrm{kink}\end{array}$ & $6 \times 10^{-5}$ & $3\left(50 \mathrm{R}_{\mathrm{E}}\right)$ & 0.001-0.002 & $\begin{array}{c}3-6 \mathrm{MeV} \\
(\text { for } Z=6 \text { ) }\end{array}$ & $\mathrm{O}^{+6} \sim 2 \mathrm{MeV}$ \\
\hline e & $\begin{array}{c}\text { earth's } \\
\text { magnetotail }\end{array}$ & $10^{-4}$ & $1\left(15 \mathrm{R}_{\mathrm{E}}\right)$ & $0.003-0.005$ & $0.9-1.5 \mathrm{MeV}$ & $\mathrm{e}, \mathrm{p} \sim 1 \mathrm{MeV}$ \\
\hline $\mathrm{f}$ & earth's foreshock & $6 \times 10^{-5}$ & $3\left(50 \mathrm{R}_{\mathrm{E}}\right)$ & |0.001-0.002 & $0.5-1 \mathrm{MeV}$ & $\mathrm{p} \sim 0.1-0.2 \mathrm{MeV}$ \\
\hline
\end{tabular}

Notes:

${ }^{1} \mathrm{CME} \equiv$ Coronal Mass Ejection. For observed $E_{\mathrm{max}} \sim 30 \mathrm{GeV}$, see Kahler (1994).

${ }^{2} \mathrm{CRAND}(=$ cosmic ray albedo neutron decay) + stochastic betatron acceleration.

${ }^{3} \mathrm{ESP} \equiv$ Energetic Storm Particles.

${ }^{4} \mathrm{ACR} \equiv$ Anomalous Cosmic Ray components.

${ }^{5} \mathrm{IMF} \equiv$ Interplanetary Magnetic Field. Freeman \& Parks (2000).

there is a close correlation between the heliospheric observations and the expectations based on the Hillas relation (1.1).

\section{Nonlinear reaction of shock acceleration}

It is expected that the acceleration process at strong astrophysical shocks is so efficient as the energy densities of accelerated particles, being comparable to the background ther$\mathrm{mal} /$ magnetic energy densities, contribute to modify the shock structure. This modification has been treated in terms of 'cosmic-ray-modified' or 'cosmic-ray-mediated' shocks (CRMS) (e.g., Drury \& Völk, 1981, Malkov \& Drury, 2001). Observational studies of 


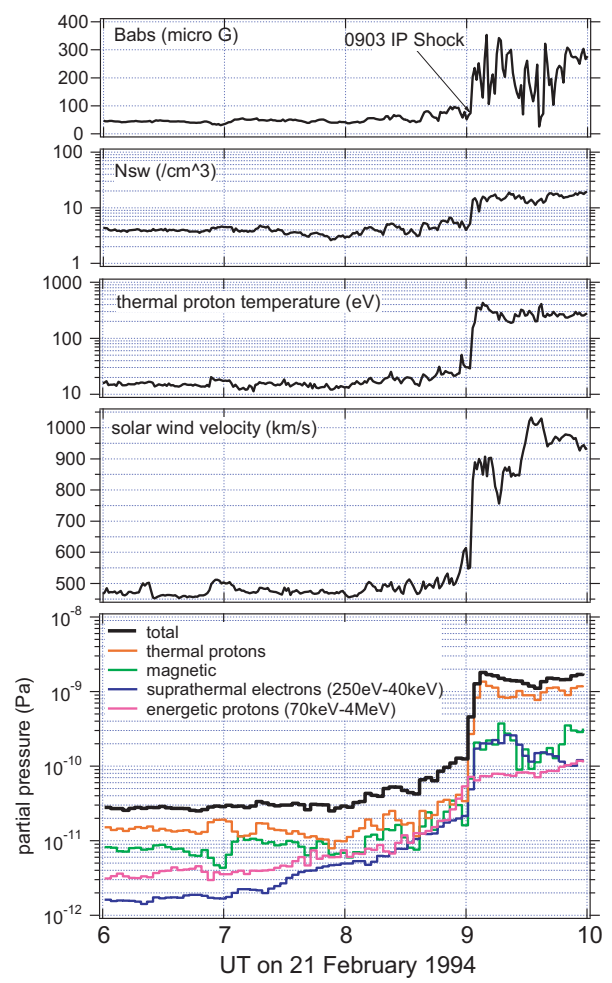

Figure 2. Observational evidence of the CRMS nature at an interplanetary shock detected at 09:03 UT on 21 February 1994. From the top panel, the magnetic field amplitude, the solar wind density, the proton temperature, the solar wind velocity, and subpressures are shown.

CRMS can be made at some of strong heliospheric shocks. Figure 2 shows an example (Terasawa, 2005, Terasawa et al., 2006), where the role of 'cosmic rays' is played by energetic protons of energy $<\sim$ several $\mathrm{MeV}$ and suprathermal electrons of energy $<\sim 40$ $\mathrm{keV}$, whose energy densities reached $\sim 15 \%$ of the upstream solar wind kinetic energy density (the bottom panel). Correspondingly, the increase of the solar wind velocity in the observer's frame $\dagger$ was seen 30 minutes- 1 hour before the arrival of the shock front itself (the fourth panel). This is an expected feature of the CRMS. Other examples of heliospheric CRMS can be found at the earth's bow shock (e.g., Zhang et al., 1995), and at the solar wind termination shock (e.g., Florinski et al. 2009. Also see the next section).

\section{Neutral particles and shock acceleration process}

It is known that the heliosphere is surrounded by the partially ionized interstellar gas with an ionization rate of $\sim 50 \%$. The neutral component of interstellar gas (mainly $\mathrm{H}$ and $\mathrm{He}$ ) can penetrate deeply into the upstream region of the solar wind termination shock. After penetration these interstellar $\mathrm{H}$ and $\mathrm{He}$ are eventually ionized either through the charge exchange process with the solar wind protons, through the photo ionization process by solar UV photons, and through the impact ionization process by energetic electrons (The third process is thought to be negligible in the outer heliosphere). Right after the ionization, these newly born ions are picked-up by the solar wind electromagnetic

$\dagger$ Decrease of the upstream plasma flow in the shock rest frame. 
field and start contributing to the solar wind dynamics importantly both in microscopic and macroscopic ways. The solar wind termination shock (TS) was crossed by Voyager 1 and 2 spacecraft successively in December 2004 and August-September 2007. For the latter crossing of TS, the plasma parameter changes were observed. At the transition layer of TS, most of the upstream solar wind flow energy is transferred not to their own thermal energy but to the interstellar pickup ions, so that the downstream solar wind ions are kept relatively cool and the flow speed after the TS crossing was supersonic (Richardson et al. 2008; Florinski et al. 2009). In this sense, TS is a 'pickup ion mediated' shock.

Interestingly, Helder et al. (2009) obtained a conclusion similar to the above TS case from the analysis of the ion temperature downstream of the shock around the supernova remnant RCW 86, which is also surrounded by the partially ionized interstellar gas. The downstream ion temperature of the RCW 86 shock is $\sim 2.3 \mathrm{keV}$ which is by a factor $\sim 18$ colder than the value expected from the Rankine-Hugoniot relation in the standard gas shock calculation, $(3 / 16) \mu m_{p} v_{s}^{2}$ with $\mu$ :cosmic abundance $(\sim 0.6), m_{p}$ :proton mass, and $v_{s}$ :shock speed $(\sim 6000 \mathrm{~km} / \mathrm{s})$. It is noted that RCW 86 is a TeV gamma ray source (Aharonian et al., 2009), where efficient particle acceleration is expected to occur. Ohira and Takahara (2010) have made a detailed calculation of the shock structure mediated by pickup ions as well as by accelerated particles, and concluded that the overall shock compression ratio can be changed significantly.

\section{Shock acceleration of electrons}

The direct measurement of cosmic ray particles at the earth shows that the electron to proton ratio is about $1 \%$ around $10 \mathrm{GeV}$. How to interpret this ratio is an important unsolved issue. Since the resonance conditions with the MHD turbulence for ions and electrons become identical above the rigidity of $\sim 10 \mathrm{GV}$, the differentiation between them should occur in the low rigidity regime where their initial accelerations occur (the 'injection' process). While the ion injection process is well understood in terms of selfexcitation of resonant MHD waves by ions themselves, how to realize the electron injection process is an unsolved issue (see, e.g., Levinson, 1992). Difficulty for the electron injection is explained in Fig. 3a: The efficient interaction between electrons and scattering waves should occur along the 'resonance line',

$$
\omega=k V_{b}-\Omega_{c e}
$$

where $\omega$ is the wave angular frequency, $k$ the wavenumber, $V_{b}$ the velocity of electrons beaming away from the shock along the background magnetic field $\left(V_{b}<0\right)$, and $\Omega_{c e}$ the electron cyclotron frequency. On this resonance line there are two interaction points, $A$ and $B$. At the point $A$ the energy exchange between electrons and whistler waves is possible, but the wave energy is absorbed by the beaming electrons, so that the wave selfexcitation is not possible. At the point $B$ the electron beam energy could be transferred to left-handed ion cyclotron waves. However, unless the electron energy is sufficiently high (namely, unless the wavenumber $k$ at $B$ is sufficiently low), thermal ions tends to suppress the wave amplification via cyclotron damping interaction.

Recent progress in the electron injection problem is made by Amano \& Hoshino (2010) who showed that at shocks with sufficiently high Alfvén Mach number $M_{A}$, whistler waves can be self-excited at the point $A$ from the loss-cone pitch angle distribution of the electrons, a natural byproduct of their shock drift acceleration. According to Amano 
(a)

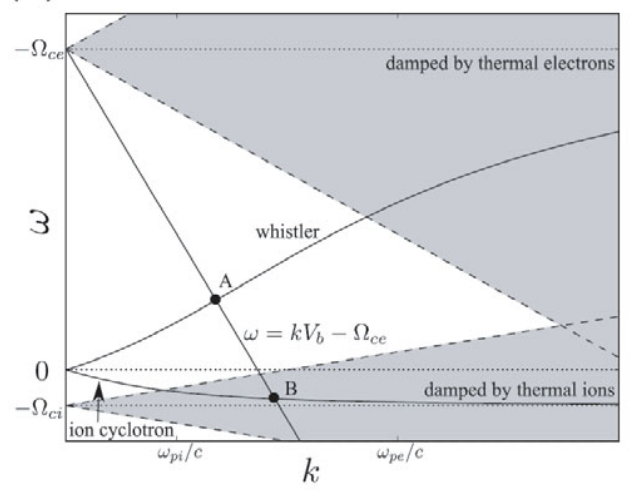

(b)

\section{Spectral Index $\Gamma$}

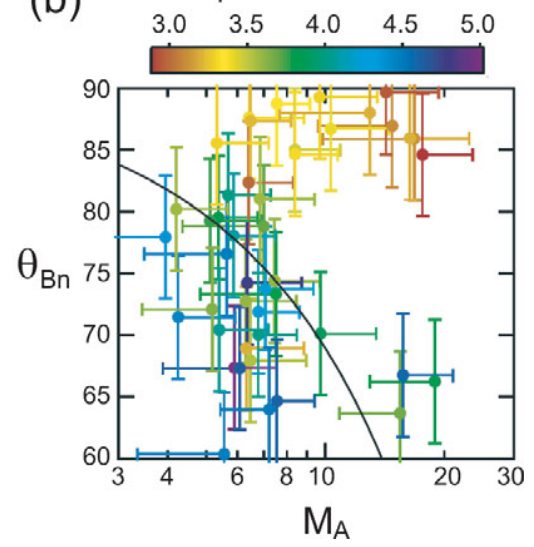

Figure 3. (a) left: Schematic dispersion diagram for circularly polarized electromagnetic waves in an electron-ion plasma, where $\omega>0(\omega<0)$ is for right-hand (left-hand) polarization. Waves in the shaded regions are strongly damped through the cyclotron resonant interaction with thermal particles (from Amano \& Hoshino (2010)). (b) right: The spectral index $\Gamma$ is obtained by fitting the power law function $E^{-\Gamma}$ to the observed electron energy spectrum $(0.05-15 \mathrm{keV})$. The observed $\Gamma$ values are shown by the color code (red-yellow-green-blue) and plotted on a $M_{A}-\theta_{B n}$ plane. A black curve shows the condition (4.2) where $\beta_{e}$ is set 1 (from Oka et al. (2006)).

\& Hoshino (2010), the condition for this mechanism can be approximated as,

$$
M_{A} \geqslant \frac{\cos \theta_{B n}}{2} \sqrt{\frac{m_{p}}{m_{e}}} \beta_{e} \equiv M_{A}^{i n j}
$$

where $\theta_{B n}$ is the angle between the shock normal direction and the upstream background magnetic field, $m_{e}$ the electron mass, $\beta_{e}$ the ratio between the electron and magnetic pressures. The authors further argue that for shocks satisfying (4.2) whistler waves grow quickly within the shock transition region and contribute to energize electrons there. This argument consistently explains the bow shock observation by Oka et al. (2006) that electrons with hard spectral index $(\Gamma<3.5)$ is mainly found when the condition $(4.2)$ is satisfied (Figure 3b).

It should be noted, however, that the whistler wave generation by the above mechanism is possible only in the region of spatial scale of a few mean free paths, beyond which electrons are isotropized and cannot excite whistler waves. For the DSA process to accelerate low energy electrons, whistler waves should spread over the entire foreshock region. Based on the observations of diffusive electron acceleration at interplanetary shocks (rare but there being a few events per one solar cycle), Nakata et al. (2003) suggested a possibility that low frequency MHD waves excited by ions nonlinearly cascade to high frequency whistler wave regime. Since the former waves are known to distribute over the entire foreshock regions (e.g., Bamert et al., 2004), such a nonlinear driving process of whistler waves, if realized, could supply the necessary electron scatterers there. Recent developments in the nonlinear theory of whistler wave turbulence (Saito et al., 2008; Gary et al., 2008; Narita \& Gary, 2010) make it possible to test Nakata's suggestion (Oka et al., in preparation). 


\section{Concluding remarks}

As described in this article, the heliosphere has been playing the role of an astrophysical laboratory for the study of collisionless shocks and relating acceleration phenomena. It is interesting to note that the historically fastest interplanetary shock seems to have arrived at the Mercury's orbit (0.3 AU) within its 'free expansion' phase (Figure 1a). For a future Mercury mission or a solar-orbiter mission, we should be ready to observe such a very fast shock environment and relating acceleration phenomena.

\section{References}

Aharonian, F., et al. 2009, Astrophys. J., 692, 1500

Amano, \& Hoshino, M. 2010, Phys. Rev. Lett., 104, 181102

Axford, I. A., Leer, E., \& Skadron, G. 1977, Proc. 15th Int. Cosmic Ray Conf., 11, 132

Bamert, K., et al. 2004, Astrophys. J. Lett., 601, L99

Bell, A. R. 1978, Mon. Not. R. Astron. Soc., 182, 147

Blandford, R. D. \& Ostriker, J. P. 1978, Astrophys. J., 221, L29

Drury, L. O. C., \& Völk, H. J. 1981, Astrophys. J., 248, 344

Florinski, V., Decker, R. B., le Roux, J. A., \& Zank, G. P. 2009, Geophys. Res. Lett., 36, L12101

Freeman, T. J. \& Parks, G. K. 2000, J. Geophys. Res., 105, 15715

Gary, S. P., Saito, S., \& Li, H. 2008, Geophys. Res. Lett., 35, L02104

Helder, E. A., et al. 2009, Science, 325, 719

Hillas, A. M. 1984, Ann. Rev. Astron. Astrophys., 22, 425

Kahler, S. 1994, Astrophys. J., 428, 837

Krymsky, G. F. 1977, Sov. Phys. Dokl., 22, 327

Levinson, A. 1992, Astrophys. J., 401, 73

Malkov, M. A. \& Drury, L. O. C. 2001, Rep. Prog. Phys., 64, 429

Nakata, K., et al. 2003, Proc. 28th Int. Cosmic Ray Conf., 6, 3697

Narita, Y. \& Gary, S. P. 2010, Ann. Geophys., 28, 597

Ohira, Y. \& Takahara, F. 2010, Astrophys. J. Lett, 721, L43

Oka, M., et al. 2006, Geophys. Res. Lett., 33, L24104

Richardson, J. D., et al., 2008, Nature, 454, 63

Saito, S., Gary, S. P., Li, H., \& Narita, Y. 2008, Phys. Plasma, 15, 102305

Schwartz, S. J. 2006, Space Science Rev., 124, 333

Smart, D. F. \& Shea, M. A. 1985, J. Geophys. Res., 90, 183

Terasawa, T. 2001, Science Tech. Adv. Materials, 2, 461

Terasawa, T. 2005, COSPAR Colloquium Ser., 16, 267

Terasawa, T., Oka, M., Nakata, K., et al. 2006, Adv. Space Res., 37, 1408

Terasawa, T. 2011, Chapter 12 of The Sun, the solar wind, and the heliosphere, IAGA special Sopron book series, 4, eds. Miralles, M. P. \& Almeida, J. S., in press.

Zhang, T. L., Schwingenschuh, K., \& Russell, C. T. 1995, Adv. Space Res., 15, 137 